\title{
GeoGebra na aprendizagem de conceitos de matemática avançada
}

Viviane Raquel Backendorf - UNIVATES / PGIE-UFRGS - vrbackendorf@ univates.br Marcus Vinicius de Azevedo Basso - PPGIE / UFRGS - mbasso@ufrgs.br

Resumo: O presente artigo consiste numa pesquisa sobre a construção do conceito de integral dupla como volume de um sólido, segundo a teoria da abstração reflexionante e o uso do software GeoGebra. A investigação em torno do assunto se deu a partir de atividades realizadas com estudantes do Ensino Superior, numa disciplina de Cálculo II. Para embasar as discussões, além da teoria da abstração reflexionante de Piaget, utilizamos a teoria desenvolvida por David Tall sobre a matemática elementar e a matemática avançada, como suporte para entender as possíveis dificuldades enfrentadas pelos estudantes no Ensino Superior. Ao analisar os resultados obtidos na pesquisa, concluímos que o uso do software Geogebra, favorece a construção de conceitos matemáticos, em especial da integral dupla como volume de um sólido.

Palavras-chave: Tecnologias Digitais, Abstração Reflexionante, Integral Dupla

\section{GeoGebra in the learning of concepts from advanced math}

Abstract: The present article consists of a research on the construction of the concept of double integral as volume of a solid, according to the theory of reflective abstraction and the use of GeoGebra software. The investigation around the subject occurred from activities carried out with students of Higher Education, in a discipline of Calculus II. To support the discussions, in addition to Piaget's theory of reflective abstraction, we use the theory developed by David Tall on elementary mathematics and advanced mathematics as a support to understand the possible difficulties faced by students in higher education. In analyzing the results obtained in the research, we conclude that the use of Geogebra software, favors the construction of mathematical concepts, especially the double integral as the volume of a solid.

Keywords: Digital Technologies, Reflective Abstraction, Double Integral

\section{Introdução}

As tecnologias digitais, que se fazem presentes em todas as áreas da educação, vêm movimentando os profissionais no sentido de aperfeiçoar sua prática para obter maior qualidade nos resultados e nos processos de ensino e de aprendizagem. No entanto, utilizar a tecnologia como um complemento de ensino, somente para verificar se há outra forma de visualizar o que fora desenvolvido, não corresponde à uma inserção que provoque a construção do conhecimento. Nesse sentido, Coll e Monereo (2010) defendem que, de nada adianta inserir a tecnologia nas aulas se não modificarmos a estrutura do ensino e, em especial, o currículo. Essa percepção e o reconhecimento de que se faz necessário inserir as tecnologias digitais nos processos de ensino e de aprendizagem, requer mudanças que podem ser confundidas como modismos ao invés de serem vistas como elementos fundamentais que contribuirão para a reconstrução do modelo atual de ensino.

Na educação matemática, por exemplo, temos à nossa disposição vários softwares de fácil utilização e que permitem a interação do estudante, possibilitando a percepção 
sobre como determinadas funções e objetos, por exemplo, podem comportar-se quando modificamos alguns elementos presentes na sua construção.

\begin{abstract}
Uma das principais contribuições das tecnologias digitais para a educação matemática foi tornar possível a "concretização" dos objetos matemáticos na tela do computador, dando a sensação de realismo e de existência material pela possibilidade de manipulação e alteração de suas propriedades. Com a possibilidade de manipular os objetos matemáticos, surge uma nova forma de pensar e fazer matemática, configurando-se uma extensão do pensamento do indivíduo (Notare e Basso, 2016, p.2).
\end{abstract}

Além dessa concretização, podemos citar também o uso da tecnologia digital como um facilitador na construção de conceitos matemáticos, através da visualização imediata e da possibilidade de modificar o objeto, podendo criá-los e recriá-los. Essa possibilidade verifica-se tanto na matemática desenvolvida na Educação Básica como no Ensino Universitário.

Como o nosso propósito é verificar se a inserção da tecnologia no Cálculo Diferencial e Integral $^{1}$, possibilita uma melhor compreensão dos conceitos, diferenciaremos, inicialmente, neste artigo, a matemática elementar desenvolvida na educação básica, da matemática avançada, cujo foco é no ensino universitário. Na sequência, abordaremos a relação entre o Cálculo e as tecnologias digitais, fundamentada por Tall. Para entender como ocorre a construção do conhecimento matemático via uso de recursos tecnológicos digitais, utilizaremos a teoria da Abstração Reflexionante de Piaget. Envolvendo os três temas elencados, apresentaremos um estudo preliminar de natureza qualitativa, realizado para verificar a contribuição da tecnologia na construção do conceito de integral dupla, analisado à luz da teoria da Abstração Reflexionante.

\title{
2. Fundamentação Teórica
}

A matemática, vista como vilã da educação, tem sido uma das disciplinas mais discutidas, e os estudos a respeito visam verificar o que leva à dificuldade de lidar com os conceitos e à incompreensão dos temas abordados. Nesse sentido, várias são as teorias que abordam o desenvolvimento do pensamento matemático, do nível elementar ao avançado. E, nesta pesquisa, utilizaremos as ideias de David Tall acerca do assunto.

Os matemáticos, segundo Tall (2002), buscam entender como funciona o pensamento matemático, para que esse entendimento possa auxiliar na melhoria da elaboração do processo de construção dos conceitos matemáticos visando aprimorar a qualidade de ensino.

Para situar o nosso tema de estudo dentro da matemática, vamos primeiramente entender o que é a matemática elementar e a avançada, bem como, o que diferencia uma da outra. A matemática elementar, segundo Tall (1995), é aquela que inicia com as percepções e ações em objetos no mundo externo, os quais são descritos e analisados conduzindo à classificação, contagem e diversas operações que correspondem a diferentes desenvolvimentos do processo matemático. A matemática avançada, por sua vez, envolve a utilização de estruturas cognitivas produzidas pela grande quantidade de atividades matemáticas que promovem a construção de novas ideias matemáticas.

Assim como Tall (1995), distinguimos a matemática elementar, onde os objetos são descritos, da matemática avançada onde os objetos são definidos. A linguagem é utilizada para formular as propriedades dos objetos, em ambas, no entanto, na matemática

\footnotetext{
${ }^{1}$ Neste texto utilizaremos o termo Cálculo para fazer referência ao Cálculo Diferencial e Integral
} 
elementar a descrição é construída a partir da experiência do objeto, enquanto na matemática avançada as propriedades dos objetos são construídas a partir da definição.

Essa inversão na construção do conhecimento matemático causa dificuldades, tornando o aprendizado mais angustiante. São perceptíveis os obstáculos que se apresentam quando um conceito novo está para ser construído, e, principalmente, quando entra na operação um objeto desconhecido. Com isso, as construções mentais não ocorrem tão naturalmente o que gera desconforto e consequente incompreensão, tornando a trajetória dos aprendizes do Cálculo repleta de impasses. E, quando não ocorre a assimilação dos conceitos, em muitos casos, o estudante opta por processos rápidos e por algoritmos mecânicos que levam à solução, mesmo que ele não tenha o entendimento do que fez.

Atualmente tem-se um recurso a mais, as tecnologias digitais, que pode auxiliar no desenvolvimento dos conteúdos matemáticos, sejam eles da matemática elementar ou avançada. Essa tecnologia vem mudando a percepção de funções e cálculo tanto no desenvolvimento cognitivo quanto no desenvolvimento dos conceitos.

Tall (1986) sugere que os conceitos de cálculo poderiam ser abordados com a utilização do computador através da construção de gráficos. O cálculo da área aproximada de uma região sob um gráfico, por exemplo, pode requerer muitos cálculos, quando feita manualmente. Já a utilização de um software dinâmico pode proporcionar uma concentração maior sobre as ideias envolvidas ao invés de investir somente nas operações. Logo, a utilização de um software matemático pode proporcionar maior exploração das possibilidades consumindo, para isto, pouco tempo. No entanto, mesmo que se tenha à disposição os recursos tecnológicos, Tall (1993) alerta que é preciso tomar cuidado ao desenvolver materiais que requerem o uso do computador e que sejam utilizados no ensino.

\footnotetext{
Um software de computador para a aprendizagem da matemática, que difere do software para fazer matemática, precisa ser projetado de forma que leve em conta o crescimento cognitivo do aluno, que pode diferir significativamente da estrutura lógica do conceito formal. Portanto, é de valor começar por considerar aspectos cognitivos relevantes para o uso da tecnologia do computador antes da principal tarefa de se concentrar em ambientes computacionais e seu papel na aprendizagem da matemática. (Tall, 1993, p.189).
}

O pesquisador, em vários momentos, destaca que não se pode pensar na utilização do computador como um instrumento, que simplesmente substitui outras formas de registro de atividades, resoluções e estratégias de resolução. Segundo Tall (2001) o uso de softwares e do computador não pode apenas sobrepor o lápis e papel por um teclado. Essa troca não acrescenta nada à construção do conhecimento, nem atinge o principal objetivo do uso do computador no ensino. O estudante, deve sim, ser estimulado e o computador deve fazer parte do processo de forma que o estudante atente para algumas características, analise e conclua a partir da interação com o ambiente computacional.

Ainda, Tall (2001) destaca a importância da utilização do computador quando este possibilita o desenvolvimento de atividades de experimentação, a partir das quais o estudante poderá observar certos fenômenos atribuindo significado. Nesse sentido, as propriedades matemáticas, por exemplo, podem ser verificadas, desenvolvidas e construídas a partir de uma atividade realizada com uso do computador. No entanto, a utilização de softwares específicos, bem como o computador, requer planejamento. E, segundo Tall (1989), a inclusão dos computadores no ensino exige mudanças nos currículos de matemática, e não somente no tipo de atividade que será realizada para desenvolver determinados conteúdos. Trata assim, das possibilidades que o computador 
oferece, mas, aponta que o significado dado aos conceitos depende da organização do currículo e da ação do professor.

Há de se reconhecer que o ambiente computacional ou softwares matemáticos podem ser úteis, auxiliando na construção de conceitos, como também, podem não agregar nada ao conhecimento. Faz-se necessário tornar mais abrangente o que se pretende que o estudante construa, pois se restringirmos o contexto, ele não avança além do que é proposto. Como cita Tall (2000), há uma grande diferença entre o cérebro humano e o computador. Enquanto o primeiro está propenso a erros, o segundo realiza algoritmos com precisão e velocidade, no entanto, o computador manipula os dados especificados por um programa criado por humano.

A utilização do computador é favorável à construção do conhecimento e de conceitos matemáticos, otimiza o tempo, possibilita a realização de conexões entre as diversas propriedades envolvidas no contexto em questão. Podemos afirmar isso baseado em várias pesquisas (Paranhos, 2000; Melo, 2002; Godarzi et al., 2009; Guimarais, 2010; Escher, 2011; Pires, 2016; Fontoura, 2016; Robotti E Frank, 2017), que procuraram verificar o quanto as tecnologias contribuem no ensino e aprendizagem da matemática e de forma especial no Cálculo.

Até aqui tratamos do Cálculo e das tecnologias. Nosso estudo, no entanto, vai além, pois pretendemos a partir dele compreender como se dá a construção do conhecimento baseado na teoria da abstração reflexionante. Essa teoria, por sua vez, mostra que é através das ações que o sujeito conhece o mundo, pois Piaget trata do conhecimento como uma construção que ocorre a partir da interação entre sujeito e objeto. O sujeito torna-se cada vez mais capaz de compreender determinadas situações através do processo de construção e reconstrução. Mesmo tendo Piaget estudado o desenvolvimento cognitivo de crianças, cujo foco é a matemática elementar, há outros pesquisadores, como Dubinsky (1991), que se baseia na teoria de Piaget para explicar vários conceitos matemáticos e construções que resultam do processo psicológico e que correspondem ao pensamento matemático avançado. $\mathrm{O}$ desenvolvimento cognitivo constitui-se como um processo contínuo de construções, dessa forma, a elaboração dos conceitos da matemática elementar é essencial na construção do pensamento matemático avançado.

Então, como se conhece? Abstraindo? Mas o que significa Abstrair? Significa arrastar, retirar, extrair, aspirar, separar, apartar. Separar mentalmente uma parte de um todo e considerar de forma separada das outras partes. Conhecimento, então, é o ato ou o efeito de abstrair a ideia ou noção de alguma coisa, logo tratamos aqui da abstração reflexionante, segundo Piaget.

Piaget (1995), distingue a 'abstração reflexionante' de uma abstração apoiada sobre objetos. Ele separa a abstração empírica da abstração reflexionante. Enquanto a primeira está relacionada com a geometria dos objetos apoiando-se sobre os objetos físicos ou sobre os aspectos materiais da própria ação como deslocamentos e atividades, a abstração reflexionante se relaciona com a geometria do sujeito.

Segundo Becker (2014, p.105) a Abstração Empírica "consiste em retirar qualidades dos objetos, ou das ações em suas características materiais, isto é, daquilo que pode ser observado." Exemplificando: ao ver uma flor, nesse caso, consideramos a flor um objeto, e ao plantar uma flor, a ação é plantar. Essa possibilidade de retirar características dos objetos ou ações é que caracteriza as abstrações empíricas.

Em se tratando da abstração reflexionante, podemos destacar que ela se dá a partir de coordenações de ações desempenhadas internamente pelo sujeito, e Piaget (1995) a classifica como aquela que se apoia sobre as formas e atividades cognitivas do sujeito, retirando delas certas características que serão utilizadas para outros fins. Da mesma 
forma, Dubinsky (1991) define a abstração reflexionante em termos de matemática avançada, como a construção de objetos mentais e de ações mentais sobre esses objetos.

Partindo dessas definições, consideramos a abstração reflexionante cada vez mais autônoma enquanto a abstração empírica avança porque se apoia na primeira. Dessa forma, dizemos que o conhecimento deriva da experiência, mas não só da experiência empírica. O sujeito não só constrói conhecimento do mundo, mas do que ele retira do objeto, das coordenações de suas ações. E, segundo Piaget (1995), as coordenações das ações do sujeito podem permanecer inconscientes, bem como dar lugar a tomadas de consciência e variadas conceituações.

\begin{abstract}
A passagem de uma coordenação, ou de muitas coordenações sintetizadas numa operação, a uma coordenação ou operação mais complexa faz-se por abstração reflexionante - que implica equilibração, por assimilações e acomodações, retirando qualidades dessas coordenações ou operações, constituindo, assim, novidades. Uma nova operação, composta de muitas coordenações de ações, mais capaz que a anterior e de maior abrangência. (Becker, 2014, p. 107).
\end{abstract}

Neste contexto, citamos a abstração pseudoempírica como um caso particular de abstração reflexionante: aquilo que o sujeito tira dos objetos são as propriedades que é capaz de introduzir neles, de acordo com o nível de suas coordenações de ações (Piaget 1995, p.147). Assim, a abstração pseudoempírica é aquela que se apoia nas propriedades dos objetos, decorrentes das modificações do objeto por ações do sujeito e melhorado pelas propriedades tiradas de suas coordenações. Ainda, em relação à abstração pseudoempírica, Becker (2014) a situa como a meio caminho entre as abstrações empíricas e reflexionantes.

Quando ocorre que o sujeito toma consciência da abstração reflexionante, podemos dizer que a mesma é uma abstração refletida. Chegamos então, ao processo de abstração reflexionante que se realiza em dois momentos: por reflexionamentos, que segundo Becker (2014), é a retirada de qualidades das coordenações que de um patamar qualquer são transferidas a um patamar superior; e, por reflexões que são as reorganizações daquilo que foi transferido a outro patamar juntamente com o que já existia. Tratamos esse processo de reflexionamentos e reflexões como um processo contínuo constituindo-se de novas construções, que são observadas em todos os estádios, pois conforme Piaget (1995), os novos patamares de reflexionamentos são construídos para que ocorram novas reflexões.

\title{
3. Procedimentos Metodológicos
}

A pesquisa em questão é de cunho qualitativo, que segundo Bogdan e Biklen (1994), configura-se de acordo com algumas características, das quais a mais evidente nesta pesquisa é aquela relacionada ao ambiente, onde valoriza-se o ambiente natural que é a fonte direta de dados e o pesquisador configura-se como o principal instrumento. $\mathrm{Na}$ investigação, o contato direto com o ambiente e com a situação é fundamental, pois existe a influência deste sobre o comportamento humano, o que é uma preocupação dos investigadores qualitativos.

Assim, as atividades que compõem esta pesquisa foram aplicadas a 49 estudantes da disciplina de Cálculo II, integrante do currículo dos cursos de Engenharia da Universidade do Vale do Taquari - UNIVATES. A aplicação ocorreu durante as aulas, seguindo o fluxo normal das mesmas. Na ementa da disciplina consta o conteúdo integral dupla, tema principal do estudo. Objetivando construir o conceito de integral dupla, organizamos esta pesquisa em duas etapas, possibilitando um acompanhamento mais adequado do processo. 
A partir da aplicação das atividades, procuramos responder à seguinte questão: "Como a tecnologia, através do software Geogebra", pode contribuir para a construção do conceito de integral dupla como volume de um sólido?"

Na primeira etapa, propomos um desafio e os estudantes tiveram que descrever suas estratégias para encontrar o volume de uma xícara, a qual não era um cilindro, nem cone, mas era semelhante a um paraboloide. Para apresentação das estratégias por parte dos estudantes não foi sugerida nenhuma fórmula matemática, nem comentou-se sobre a possibilidade de utilização da integral. Vale lembrar que, em aulas que antecederam essa pesquisa, os estudantes resolveram cálculos utilizando a integral de funções de uma variável e de duas variáveis, inclusive utilizaram a integral definida para calcular a área de uma determinada região.

Na segunda etapa, utilizamos um applet do Geogebra ${ }^{3}$ para verificar o volume de um sólido a partir da decomposição desse sólido em prismas retangulares. Nosso objetivo, a partir da atividade e interação com o applet foi a construção do conceito de integral dupla como volume de um sólido.

Para isso, iniciamos inserindo uma função no campo de entrada do applet, resultando na visualização em 3D. De acordo com o applet, o paraboloide é preenchido por prismas retangulares, cuja soma dos volumes tende a um determinado valor, que corresponde ao volume do sólido construído, o paraboloide.

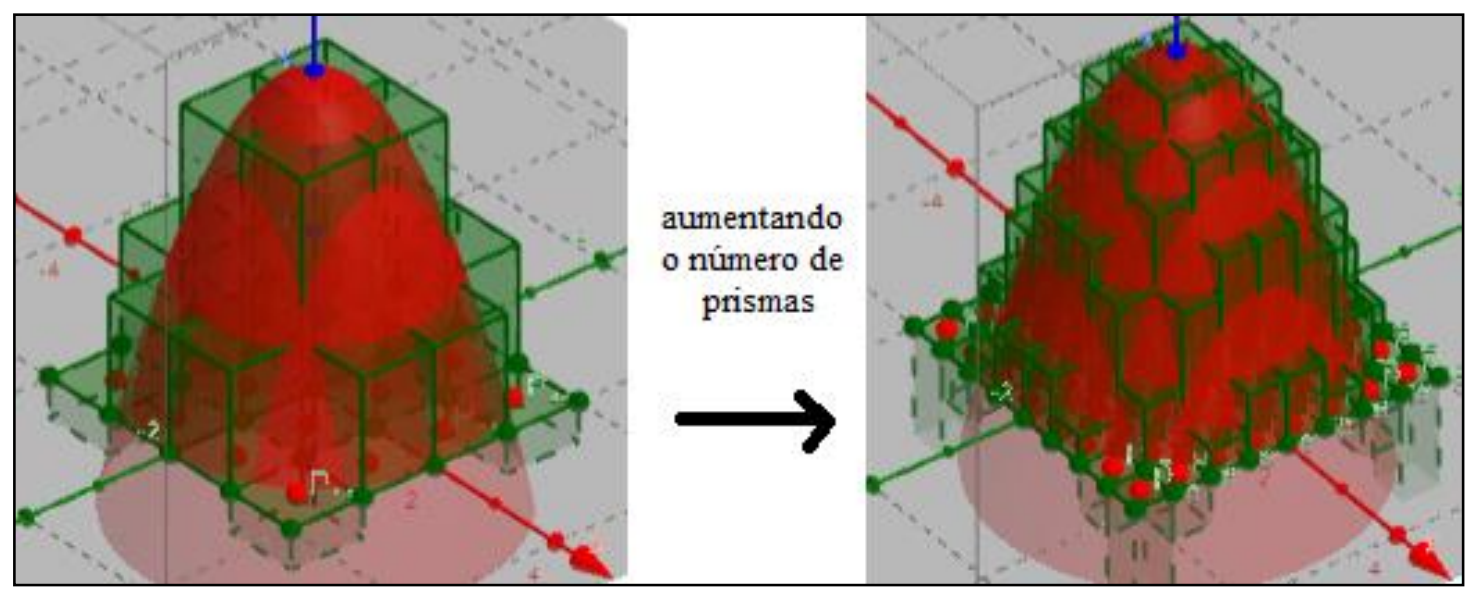

Figura1- Prismas retangulares preenchendo o sólido, paraboloide Fonte: do autor

À medida que diminuímos as medidas da base de cada prisma, aumentamos a quantidade de prismas, cujas alturas respeitam o limite superior, dado pela função do paraboloide construído. A partir da interação com o applet do software é possível perceber que a soma dos volumes dos prismas se aproxima cada vez mais do volume do sólido, neste caso, o paraboloide.

\section{Resultados e Discussão}

Diante do desafio de calcular o volume de uma xícara, os estudantes apresentaram diferentes estratégias de resolução sendo que a decomposição da xícara em diferentes sólidos foi a estratégia mais frequente. Para fazer uma análise do processo, e verificar os

\footnotetext{
${ }^{2}$ O software Geogebra, é um software de matemática dinâmica gratuito, criado como tese por Markus Hohenwarter em 2001, na University of Salzburg. O software combina geometria, álgebra, tabelas, gráficos, estatística e cálculos numa única aplicação.

${ }^{3}$ Applet criado por Waldecir Bianchini, disponibilizado: www.geogebra.org/u/waldecir\#materials/created integral dupla - https://www.geogebra.org/m/fqJ2PkAS
}

V. $16 \mathrm{~N}^{\mathrm{o}} 1$, julho, 2018 
detalhes na resolução das atividades, selecionamos cinco estudantes, cujas estratégias apresentamos abaixo:

Estudante A: "Eu utilizaria a fórmula do cone diminuindo: raio maior - raio menor e dividiria por três."

Estudante B: "Calcularia o volume de um cilindro (interno) e um cone (externo). Somaria os dois."

Estudante C: "Utilizando a fórmula: $V=\frac{\pi h}{3}\left[R^{2}+R r+r^{2}\right]$, onde $h=$ altura, $R$ = raio maior do círculo superior, $r=$ raio menor do círculo inferior. Outra forma de analisar seria, encher a xícara com água e pesar. Esvaziar ela e novamente pesar. Por fim, subtrair os valores para achar o volume."

Estudante D: "O volume de um cilindro normal é dado por $V=\pi r^{2} h$, o cone será $\frac{1}{3}$ do volume desse cilindro e teremos duas bases de raios diferentes. Logo, substituindo $V=\frac{1}{3} \pi h\left[R^{2}+R r+r^{2}\right]$ "

Estudante E: "Calcularia a integral do volume da xícara: $V=\pi r^{2} \int \pi r^{2} h$ "

Verificamos que os estudantes citados utilizaram os conhecimentos anteriormente construídos sobre o volume de sólidos. Para calcular o volume procuraram entre os sólidos que mais se assemelham à xícara, como o cone, cone truncado e cilindro. Um estudante mencionou a utilização da integral, no entanto, não soube argumentar como essa integral poderia ser utilizada.

Nas estratégias apresentadas pelos estudantes, verificamos uma abstração empírica, que segundo Piaget (1995), "é a abstração que se apoia nos objetos", pois buscaram a solução utilizando sólidos conhecidos, os quais possivelmente já manipularam para encontrar o volume. Percebeu-se a presença de abstração pseudoempírica pelas propriedades de alguns sólidos, consideradas para calcular o volume da xícara. Observamos também, uma busca por conhecimentos e conceitos prévios, e, a descrição do cálculo do volume se deu a partir da experiência do objeto, que segundo Tall (1995) é característica da matemática elementar. A sugestão de utilização da integral poderia ser interpretada como uma estratégia partindo de definições, o que abordaria a matemática avançada, conforme Tall (1995), no entanto, interpretamos a utilização da integral pelo estudante como uma alternativa, já que o conteúdo que estava sendo desenvolvido na disciplina era integral.

Na etapa seguinte, observamos que a utilização das tecnologias digitais através do applet do software Geogebra proporcionou um olhar diferente para um sólido, cujo cálculo do volume não era trivial. Através da interação com o applet, foi possível verificar que a soma dos volumes dos prismas retangulares tendia a um limite que seria o volume do paraboloide. Esse limite, por sua vez, podia ser interpretado como a integral dupla da função desse sólido no intervalo dado. Questionados sobre a soma dos volumes dos prismas, cuja quantidade podia ser aumentada ou reduzida, os cinco estudantes responderam que aquele volume diminuiria até um certo valor, que correspondia ao volume do sólido construído com a inserção da função $f(x, y)=4-x^{2}-y^{2}$, no applet. Cabe ressaltar que, quanto maior a quantidade de prismas, menor a base de cada um deles.

Ao serem questionados sobre os intervalos definidos para o sólido construído no applet, afirmaram que a partir dos intervalos tornava-se possível calcular o volume do sólido através do cálculo da integral dupla, cujo resultado confrontado com a soma dos volumes dos prismas, era o mesmo, ou então, ficaria muito próximo. Ao responderem dessa forma, observamos que os estudantes apresentaram uma abstração pseudoempírica, pois utilizaram o conceito de limite para dirimir sobre o volume do sólido. Além disso, verificamos a presença da abstração reflexionante, pois traduziram todas as ações e conceitos envolvidos como um único: integral dupla. 
De acordo com a construção de conceitos matemáticos avançados, defendida por Tall (1986, 1993, 2001), deve ser dada a devida importância à utilização do computador, pois possibilita a experimentação. Percebemos, assim, que os estudantes aproveitaram esquemas próprios, e os confrontaram com a atividade realizada no applet do software Geogebra, quando promoveram ações em objetos como a representação gráfica da função, verificação do volume dos prismas retangulares e o volume do sólido construído a partir da inserção da função. Com isso, observamos que a coordenação de ações desse processo levou a novos processos e enfim a uma generalização, que segundo Piaget (1995) é o resultado de uma abstração reflexionante. Isso, devido às relações que estabeleceram entre a soma dos volumes dos prismas e a integral dupla da função do sólido, cujo volume pretendia-se encontrar. Assim, consideramos que ocorreu a construção do conceito de integral dupla como volume de um sólido.

Os estudantes, em entrevista sobre o uso do applet na construção do conceito de integral dupla, afirmaram:

Estudante A: "O software contribui, pois possibilita que tenhamos uma visualização do objeto e assim podemos observar como a integral funciona dentro dele."

Estudante B: "O applet do software geogebra contribui no cálculo do volume do sólido, dando através do preenchimento de blocos sob o sólido, um valor cada vez mais próximo entre prisma (quando aumentados $m$ e $n$ ) e sólido. Manualmente, calculando bloco por bloco - prismas, obteríamos também o volume do prisma, mas demoraria muito. O volume do sólido seria descoberto com maior facilidade pelo cálculo da integral dupla."

Os estudantes A e B, a partir das afirmações mostraram abstração empírica, pois basearam-se na visualização do objeto para concluir sobre o volume. E, ao associar a integral dupla ao volume, apresentaram abstração reflexionante.

Estudante C: "O geogebra ajudou na visualização dos limites, e do cálculo do volume, através da soma dos volumes dos sólidos de base retangular enquanto a mesma ia diminuindo. Observou-se uma semelhança nos resultados do applet e do cálculo feito pela integral dupla. Concluímos que a integral dupla é uma ferramenta que possibilita o cálculo do volume dos sólidos, através da função e seus limites."

Estudante D: "O software contribui na visualização do sólido e com a utilização dos prismas, nos aproximamos do valor real do volume. Vimos que manualmente podemos utilizar a integral dupla para calcular o volume."

Estudante E: "No geogebra tu consegue sobrepor os prismas no paraboloide aproximando o volume da medida real do sólido. Se fizéssemos manualmente, conseguiríamos um resultado aproximado, mas seria muito cansativo e com margem de erro muito grande. Assim, teríamos que usar a integral dupla que é o volume de um sólido."

A generalização verificada nas afirmações desses estudantes atestou que o processo de construção no applet possibilitou uma abstração reflexionante.

Verificamos que o applet auxiliou na descoberta do volume do paraboloide e ao mesmo tempo, levou à construção do conceito de integral dupla, que foi definida como alternativa para calcular o volume de um sólido manualmente.

Depois da realização de atividades com utilização do software geogebra, os estudantes retornaram ao desafio da xícara. Modelaram a mesma, inserindo funções no campo de entrada do geogebra, de acordo com as medidas dadas. Baseados na atividade do paraboloide, partiram da função $f(x)=4-x^{2}-y^{2}$ e foram modificando a mesma, de forma que o sólido ficasse o mais próximo possível da xícara. Observando os estudantes, durante a realização do desafio, percebemos o quanto o software contribuiu para encontrar a função mais adequada. A partir da função e dos intervalos atribuídos ao sólido, os 
estudantes, de acordo com os conceitos construídos, calcularam o volume da xícara utilizando a integral dupla. $\mathrm{O}$ fato de resolverem um problema de volume utilizando a integral dupla deixou evidente a contribuição da tecnologia digital na construção desse conceito, demonstrou uma abstração reflexionante, pois a partir das atividades realizadas, os estudantes optaram pelo cálculo da integral de uma função num determinado intervalo para verificar o volume desse sólido.

\section{Considerações Finais}

No contexto desta pesquisa, procuramos verificar se a utilização das tecnologias digitais propicia os processos de abstração reflexionante. Os dados apresentados neste estudo sugerem uma afirmação positiva para esse questionamento, pois identificamos a ação do sujeito sobre os recursos do software, produzindo observáveis do objeto, cuja reorganização contribui para a reorganização do pensamento levando às abstrações por reflexionamentos e reflexões.

Assim, a teoria da abstração reflexionante de Piaget contribuiu para analisar como o estudante constrói o conhecimento e os conceitos matemáticos, pois em diferentes momentos e atividades a presença da abstração empírica, pseudo-empírica e reflexionante corroborou esta afirmação.

Analisando os resultados obtidos, os autores pretendem em investigações futuras, realizar um estudo mais individualizado, com menor quantidade de sujeitos, para verificar de forma mais pontual a utilização e construção de conceitos da matemática avançada. E, assim, analisar de forma mais intensa cada uma das etapas na construção do conceito de integral dupla, valorizando todo o processo.

\section{Referências Bibliográficas}

BECKER, Fernando. Abstração pseudo-empírica e reflexionante: Significado epistemológico e educacional. In: Schème - Revista Eletrônica de Psicologia e Epistemologia Genéticas. Vol. 6. UNESP, 2014. Disponível em: www.marilia.unesp.br/scheme Acesso em 16 de agosto 2016.

BIANCHINI, W. Integral dupla - software Geogebra. Disponível em: www.geogebra.org/u / waldecir\#materials/ created - www.geogebra.org/m/fqJ2PkAS. Acesso em: 20/ago/ 2017.

BOGDAN, R. C e BIKLEN, S. K. Investigação Qualitativa em Educação. Tradução de Maria João Alvarez, Sara Bahia dos Santos e Telmo Mourinho Baptista. Porto - Portugal: Porto Editora, 1994.

COLL, C., MONEREO, C. Psicologia da educação virtual: aprender e ensinar com as tecnologias da informação e da comunicação. Porto Alegre, Artmed, 2010.

DUBINSKY, Ed. Reflective Abstraction in Advanced Mathematical Thinking. In: D. Tall (Ed.) Advanced Mathematical Thinking, Kluwer: Dordrecht, pp. 95-123, 1991. Disponível em: http://www.math.wisc.edu/ wilson/Courses/Math903/ReflectiveAbstraction. Acesso em 10 de novembro de 2017.

ESCHER, M. A. Dimensões Teórico-Metodológicas do Cálculo Diferencial e Integral: perspectivas histórica e de ensino e aprendizagem. São Paulo. Rio Claro: UNESP, 2011. 222p. Tese de Doutorado. Disponível em: http://bancodeteses.capes.gov.br /banco-teses/\#!/. Acesso em 25 de agosto de 2017.

FONTOURA, L. R. Uma sequência de ensino para o estudo de integrais duplas. Rio Grande do Sul. Santa Maria. Centro Universitário Franciscano. 2016. Dissertação de Mestrado em Ensino de Matemática. Disponível em: http://bancodeteses.capes.gov.br /banco-teses/\#!/. Acesso em 25 de agosto de 2017. 
GODARZI, S. Q.; BAKHSHALIZADEH, S.; AMINIFAR, E. The impact of using computer Algebra Systems (CAS) in teaching and learning of "Double Integral”. Irã, 2009.

GUIMARAIS, Y. P. B. de Q. Exploração de convergência em tópicos de cálculo diferencial, integral e numérico, usando os softwares VCN e Geogebra. Minas Gerais. Belo Horizonte, PUC-MG. 2011. 192p. Dissertação de Mestrado. Disponível em: http://bancodeteses.capes.gov.br /banco-teses/\#!/. Acesso em 25 de agosto de 2017.

MELO, J. M. R. Conceito de Integral: uma proposta computacional para seu ensino e aprendizagem. São Paulo. PUC-SP, 2002. Dissertação de Mestrado. Disponível em: http://bancodeteses.capes.gov.br /banco-teses/\#!/. Acesso em 25 de agosto de 2017.

NOTARE, M. R.; BASSO, M. V. A. Geometria Dinâmica 3D - novas perspectivas para o pensamento espacial. RENOTE: Revista Novas Tecnologias na Educação, Porto Alegre, v.14, n. 2, dezembro, 2016. Disponível em: http://seer.ufrgs.br/index.php/renote/article/ view/ 70683. Acesso em 16 de junho de 2017.

PARANHOS, M. M. Geometria Dinâmica e o Cálculo Diferencial e Integral. São Paulo. PUC-SP, 2000. 112p. Dissertação de Mestrado. Disponível em: http://bancodeteses.capes.gov.br /banco-teses/\#!/. Acesso em 25 de agosto de 2017.

PIAGET, Jean. Abstração reflexionante: relações lógico-aritméticas e ordem das relações espaciais. Tradução: Fernando Becker e Petronilha Beatriz Gonçalves da Silva. Porto Alegre: Artes Médicas, 1995.

PIRES , L. F. R.. As Influências das Tecnologias da Informação e Comunicação nas Estratégias de Ensino e Aprendizagem de Cálculo Diferencial e Integral. Minas Gerais. Universidade Federal de Juiz de Fora, 2016. Dissertação de Mestrado Profissional. Disponível em: http://bancodeteses.capes.gov.br. Acesso em 17 de setembro de 2017.

ROBOTTI, E.; FRANK, B. Using Digital Environments to Address Students' Mathematical Learning Difficulties. In: Innovation and Technology Enhancing Mathematics Education Mathematics Education in the Digital Era, FAGGIANO, E.; FERRARA, F.; MONTONE, A.

(Ed). Itália: Springer, 2017, p. 77-106.

TALL, D. O. A graphical approach to integration and the fundamental theorem. Mathematics Teaching, 113 48-51, 1986.

TALL, D. O. Computer environments for the learning of mathematics. Didactics of Mathematics as a Scientific Discipline - The State of the Art, ed R. Biehler, R. Scholtz, R. W. Sträßer, B. Winkelmann. Dordrecht: Kluwer, 189-199, 1993.

TALL, D. O. Mathematical Growth in Elementary and Advanced Mathematical Thinking, plenary address. In L. Meira \& D. Carraher, (Eds.), Proceedings of PME 19, Recife, Brazil, I, 61- 75, 1995.

TALL, D. O. Cognitive Development in Advanced Mathematics Using Technology. Mathematics Education Research Journal. 12 (3), 196-218, 2001.

TALL, D. O. The Psychology of Advanced Mathematical Thinking. In Advanced Mathematical Thinking (Vol. 11, pp. 3-21). Kluwer Academic Publishers, 2002. 